\title{
Search For Cool White Dwarf Pulsators
}

\author{
Atsuko Nitta ${ }^{1}$, A. Mukadam ${ }^{1}$, D. E. Winget ${ }^{1}$, A. Kanaan ${ }^{2}$, \\ S. J. Kleinman ${ }^{3}$, S. O. Kepler ${ }^{4}$, M. H. Montgomery ${ }^{5}$
}

\begin{abstract}
We are searching for pulsations in cool $(<6000 \mathrm{~K})$ white dwarfs (WDs), hoping to apply asteroseismological techniques to improve our understanding of their structure and the physical processes inside them. This information is important as we use cool WDs to estimate the lower limit of the age of the Galactic disk. Within a spectroscopic and photometric survey of 110 cool WDs by Bergeron, Ruiz, \& Legget, we find 28 candidates with appropriate effective temperatures, masses, and chemical compositions for possible pulsations in nonradial $\mathrm{g}$ modes with periods similar to those we observe in DAVs. So far, we have observed 4 candidates, but have found no evidence of large variation.
\end{abstract}

\section{Goal of the Project}

We use white dwarfs (WDs) as reliable and independent chronometers to estimate the lower limit of the age of the Galactic disk via the observed turn down of the WD luminosity function (WDLF) (Winget et al. 1987), and as laboratories for the behavior of matter at pressures and densities unachievable on earth. The largest uncertainty in the WDLF lies in our understanding of the structure and physics, especially the crystallization process, of the cool WDs. If we are able to find pulsating cool WDs, then by the techniques of asteroseismology, we can extract the physical parameters for the WDs directly contributing to the downturn in the WDLF.

Historically, we have found the instability strips of the WDs to be associated with the partial ionization of chemical elements. For the DAVs, this element is $\mathrm{H}$, and for the DBVs, it is He (Dolez \& Vauclair 1981; Winget et al. 1982a,b). Recently, Stobie et al. (1997) have found pulsations in sdB stars which Charpinet et al. (1996) predicted to exist from the opacity bump associated with heavyelement ionization. For the very cool WDs, molecular states start to become important sources of opacity in their atmospheres. Thus a "partial dissociation"

\footnotetext{
${ }^{1}$ Department of Astronomy, University of Texas at Austin, Austin, TX 78712, U.S.A.

${ }^{2}$ Universidade Federal de Santa Catarina, Campus Universitario, Trindade, CEP 88040-900, Florianopolis, SC, Brazil

${ }^{3}$ Department of Physics \& Astronomy, Iowa State University, Ames, Iowa 50011, U.S.A.

${ }^{4}$ Universidade Federal do Rio Grande do Sul, 91501-900 Porto Alegre, RS Brazil

${ }^{5}$ Institut für Astronomie, Universität Wien, Tuerkenschanzstrasse 17, A-1180 Wien, Austria
} 
region might exist in terms of the bound and unbound molecular states, which might drive nonradial g modes. This partial dissociation zone would be farther out in the envelope than the $\mathrm{H}$ ionization zone, so the shorter thermal time scales in this region could lead to the driving of pulsations with periods perhaps as short as those seen in the DAVs. Another source which can modulate the luminosity inside the WDs is the process of crystallization. The crystal/fluid interface moves outwards in the WDs as they cool. If this interface region is far enough out in the WD, it might be able to drive nonradial $g$ modes. For both of these reasons, we believe that cool WDs may pulsate (see Winget 1998).

\section{Observations and Preliminary Results}

All of our candidates (from Bergeron, Ruiz, \& Legget 1997) either have above $90 \%$ of crystallization mass fraction according to Wood's (1990) WD models, or show $\mathrm{C}_{2} \mathrm{H}$ features. We observed 4 candidates using a CCD on the 2.1-m telescope at McDonald observatory in 1998 September. These cool WDs may have similar periods to the known DAVs, in the range of 100 to $1000 \mathrm{~s}$. Our cycle time was around $80 \mathrm{~s}$ and we used a $V$ filter to minimize the color differences between our targets and the comparison stars. We observed each target for 4 hr twice to guard against the case in which beating has reduced the amplitudes below our detection limit.

The four targets we observed so far show no signs of pulsation to an amplitude limit of 1 to $3 \mathrm{mma}$ ('milli modulation amplitude'). In view of the potential importance of finding an extremely cool pulsating WD, we will continue this project at McDonald Observatory, and at Kitt Peak Observatory in the future.

\section{References}

Bergeron, P., Ruiz, M. T., \& Legget, S. K. 1997, ApJS, 108, 339

Charpinet, S., Fontaine, G., Brassard, P., \& Dorman, B. 1996, ApJ, 471, L103

Dolez, N. \& Vauclair, G. 1981, A\&A, 102, 375

Stobie, R. S., Kawaler, S. D., Kilkenny, D., O’Donoghue, D., \& Koen, C. 1997, MNRAS, 285, 651S

Winget, D. E., Tassoul, M., Fontaine, G., \& Hansen, C. J. 1982a, ApJ, 251, L65

Winget, D. E., Robinson, E. L., Nather, R. E., \& Fontaine, G. 1982b, ApJ, 262, L11

Winget, D. E., Hansen, C. J., Liebert, J., Van Horn, H. M., \& Fontaine, G. 1987, ApJ, 315, L77

Winget, D. E. 1998, Journal of Phys.: Condens. Matter, 10, 11247

Wood, M. A. 1990, PhD Thesis, University of Texas at Austin 